\title{
Tune variation and transverse displacement as a diagnostic for profiling beam energy
}

\author{
B. Beaudoin and R. A. Kishek \\ Institute for Research in Electronics and Applied Physics, University of Maryland, College Park, Maryland 20742, USA
}

(Received 22 January 2012; revised manuscript received 14 October 2013; published 18 November 2013)

In this paper we describe two methods that demonstrate an energy evolution at the ends of a long bunch. The first method is a nondestructive diagnostic that extends the response matrix technique, to provide insight into the time-sliced transverse behavior of a coasting bunch in a ring. This method uncovers intense beam physics from the resulting longitudinal energy variations at the ends of a rectangular bunch. Measurements of the tune profile, using this method, agree with analytical predictions resulting in a percent error of $0.32 \%$ and $2.45 \%$ (head and tail respectively). Measurements and calculations presented in this paper are presented for a beam with an intensity of 0.901 (beam current of $21 \mathrm{~mA}$ ). The head and the tail tunes, averaged over four ring chambers, also illustrate the intensity variation axially along the bunch. This is exhibited through tune-shift measurements at the ends of the bunch. As confirmed through calculations, the measured tune shift at the head is smaller than the tail. This results from the sum of both the transverse coherent tune shift from image forces with the component from longitudinal space charge. The sum of the two components produces a smaller tune shift at the head of the beam and a larger tune shift at the tail. The second method presented in this paper optically illustrates the centroid "cork-screwing" motion within the beam ends, at a single location on the ring. The motion is resolvable using this diagnostic since it is capable of axially time-slicing regions of the beam with different energies. The difference between the two methods presented is that the first is a nondestructive diagnostic that can be extended to multiple turns whereas the second method is a destructive first turn imager.

DOI: 10.1103/PhysRevSTAB.16.114201

PACS numbers: 52.59.Sa, 41.75.-i, 29.27.Bd

\section{INTRODUCTION}

Accelerator research has seen a recent shift towards an emphasis on increasing beam intensity or luminosity rather than just energy [1]. This shift has resulted in the increased importance of considering the impact of space charge, as well as other sources of emittance growth that can limit beam intensity [2]. Achievable luminosities can often be limited by beam behavior at the low-energy end of the machine, even for accelerator systems where space charge and emittance growth are negligible for the bulk of the accelerator.

The longitudinal self-forces in these beams are continually evolving. Any externally applied focusing forces must evolve accordingly for the beam to remain in a local equilibrium. This is to avoid the excitation of longitudinal space-charge waves and degradation of beam quality $[3,4]$. Applying the necessary waveforms needed to maintain the shape of these intense beams requires knowledge of both the current and velocity distribution of the beam, since these characteristics determine the wave structure.

Induction accelerators, in particular linear accelerators, are able to attain high intensities by utilizing wideband induction modules $[4,5]$ that manipulate the longitudinal velocity distribution along the beam. The neutralized drift

Published by the American Physical Society under the terms of the Creative Commons Attribution 3.0 License. Further distribution of this work must maintain attribution to the author(s) and the published article's title, journal citation, and DOI. compression experiment (NDCX-II) at Lawrence Berkeley National Laboratory is currently planning to longitudinally confine, compress, and accelerate an intense ion beam in order to obtain a subnanosecond pulse for warm dense matter (WDM) and inertial fusion energy (IFE) studies. To accomplish this, the pulsed waveforms in each of the induction modules are tailored to achieve a complex timing schedule derived from simulations. Experimental diagnostics will measure current, energy, and transverse profiles to refine the simulation models.

While a relatively short machine like NDCX-II represents a significant milestone in the study of intense beams for IFE; a much longer full-scale higher energy machine is required. Construction of a long full-scale experiment will require a substantial amount of time before it can access some of the novel physics, like wave reflection. A promising approach to exploring this area of physics has been demonstrated in the University of Maryland Electron Ring (UMER) which uses a scaled ring topology to access the physics of intense beams over long path lengths. UMER has demonstrated the ability to access tens of kilometer scale propagation lengths $\left(>3 \times 10^{4}\right.$ alternating gradient periods), whereas current linear machines have generally been limited to hundreds of meters in length.

UMER is a scaled high-intensity circular induction accelerator dedicated to the study of long path-length transverse and longitudinal space-charge-dominated beam physics using electrons. The typical UMER beam has an average energy of $10 \mathrm{keV}, 1 \mu \mathrm{m}$ transverse emittance, and 
a variable current in the range of $0.5-100 \mathrm{~mA}$. A long (100 ns) rectangular beam is injected into the ring that fills approximately half the circumference. We then allow the bunch to elongate axially by disabling the longitudinal containment fields (rf). The ring also contains multiple diagnostics that measure position, current, and beam size over many turns. Though an electrostatic energy analyzer capable of measuring energy profiles is pending, it is a destructive first turn diagnostic [6].

In this paper, we report on two diagnostics that demonstrate an energy evolution at the ends of a long bunch. The first diagnostic extends the response matrix technique to provide insight into the time-sliced transverse behavior of a coasting bunch in a ring. This diagnostic, capable of being extended to multiple turns, uncovers intense beam physics from the resulting longitudinal energy variations at the ends of a rectangular bunch. We also illustrate the phenomena at the ends, using a destructive first turn diagnostic that images the "cork-screwing" motion. With these two diagnostic tools, we have been able to infer the basic shape of the longitudinal energy profile along the bunch. In Sec. II we present a summary of the predictions from a one-dimensional cold fluid model, describing essential features of beam elongation. This includes calculations of the energy gained and lost at the head and tail as well as simple calculations of the correlated tune along the beam. The calculations also include the coherent contribution due to image forces. In Secs. III and IV, we describe measurements of betatron tune and transverse centroid displacement along the beam, as the bunch freely expands within the first turn from space charge and the absence of longitudinal focusing. Section V discusses the results and conclusions, including potential methods for expanding the first measurement to a multiturn diagnostic for the longitudinal containing fields.

\section{LONGITUDINAL BEAM EROSION IN A RING}

The longitudinal dynamics of a space-charge-dominated beam can be adequately characterized using the onedimensional cold fluid theory [7]. This is a valid approximation in the long wavelength regime since the beam is found to have a very low longitudinal thermal velocity from accelerative cooling in the source and a length that is long compared to the pipe radius [2,8]. If a beam is injected with constant charge line density and constant velocity, self-fields will push particles in the beam ends away from the central region. This will cause both ends of the beam to expand, in the beam frame, at a rate of $2 c_{s}$; where $c_{s}$ is the longitudinal space-charge wave velocity, $c_{s}=\sqrt{q g \lambda_{o} / 4 \pi \varepsilon_{o} \gamma_{o}^{5} m}, q$ is the electron charge, $m$ the electron mass, $\gamma_{0}$ the Lorentz factor, $\varepsilon_{0}$ the permittivity of free space, and the variable $g$ is the geometry factor accounting for the pipe shielding of the longitudinal self-fields [7-10]. One caveat of this simple model is that it neglects the variation in $g$ factor at the beam ends, but it has been found experimentally to be an adequate approximation [4].

The expansion is also accompanied by a rarefaction of the beam ends, eroding into the beam at a rate of $c_{s}$ (an illustration is shown in Fig. 1).

Figure 1 illustrates the charge line density and energy profile as a function of time. The profile starts from an initial rectangular beam (shown in blue) distribution at $s=0$ and ends at a distance $s=s_{1}$ (shown in black) [2,7]. A particle at the leading edge of the beam head undergoes an energy gain of $\Delta E=2 m c_{s}\left(\mathrm{v}_{o}+c_{s}\right)$. The leading edge of the beam head occurs where the charge line density goes to zero. The opposite is true for a particle at the tail. A particle at the leading edge of the beam tail undergoes an energy loss of $\Delta E$. This has been shown using a destructive electrostatic energy analyzer on a short linear experiment [11].

A schematic diagram of the ring, including ring chambers $9,11,13$, and 15 (RC9, $\mathrm{RC} 11, \mathrm{RC} 13$, and $\mathrm{RC} 15)$ is displayed in Fig. 2.

The primary diagnostics employed to measure transverse position are the beam position monitors (BPMs) located at RC9, RC11, RC13, and RC15 as well as a fast phosphor screen located at RC15. The nominal machine and beam parameters for the experiments presented in this paper are listed in the Appendix.

The current and velocity profiles, calculated from the solutions to the cold fluid equations, are shown below as a function of time within the first turn (Fig. 3). The profiles are calculated from the source up to the location of RC9 $(7.02 \mathrm{~m}), \mathrm{RC} 11(8.30 \mathrm{~m}), \mathrm{RC} 13(9.58 \mathrm{~m})$, and $\mathrm{RC} 15$ (10.86 m).

The current and velocity profiles illustrate the elongation of the bunch. The head of the beam at RC9 is $7.8 \mathrm{~ns}$ in length. The same is true for the length of the tail at RC9. As

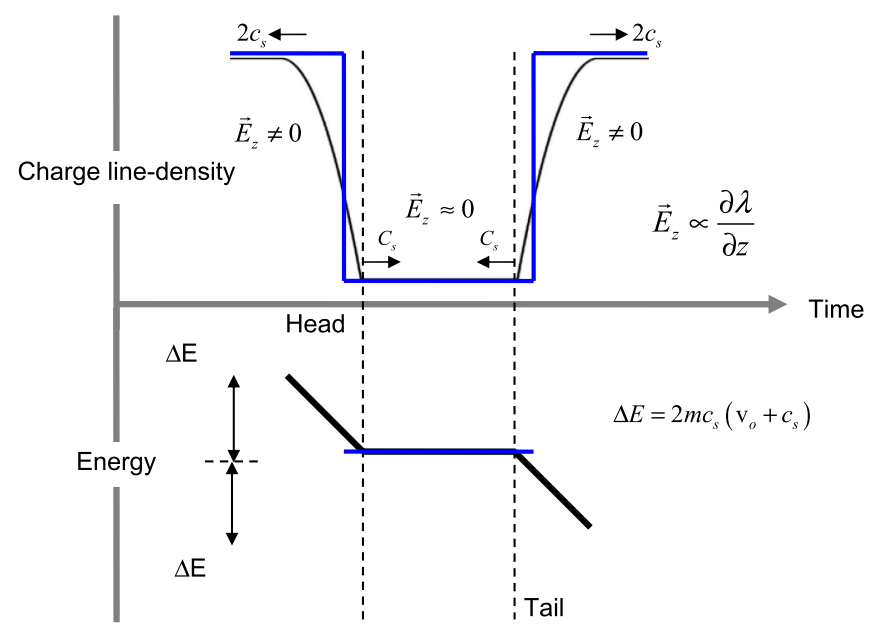

FIG. 1. Beam charge line density and energy profile snapshot illustrations as a function of time, starting from an initially rectangular beam distribution at $s=0$ (shown in blue). The curves in black are the same as in blue but at a distance $s=$ $s_{1}$ from the source. 


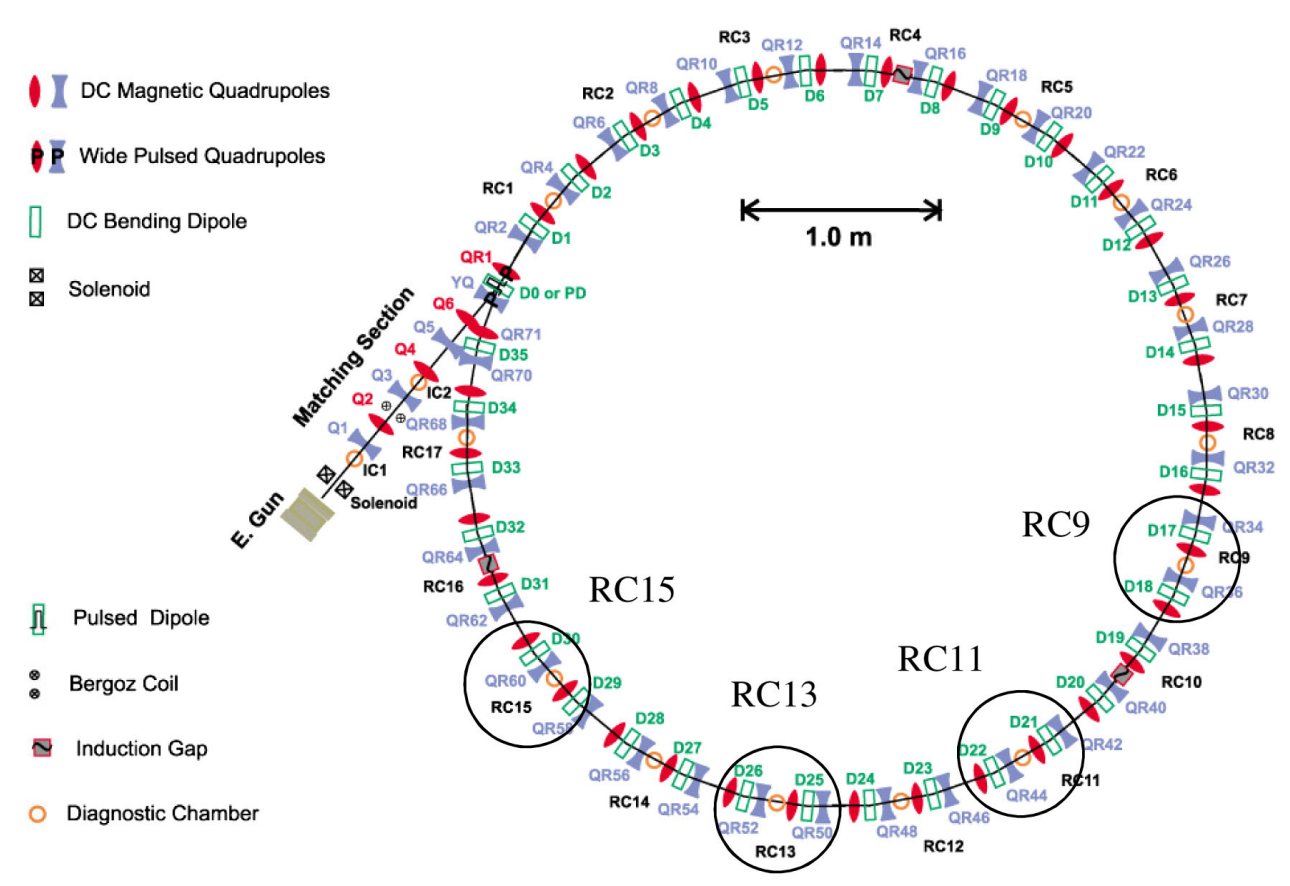

FIG. 2. Lattice optics diagram with RC9, RC11, RC13, and RC15 circled.
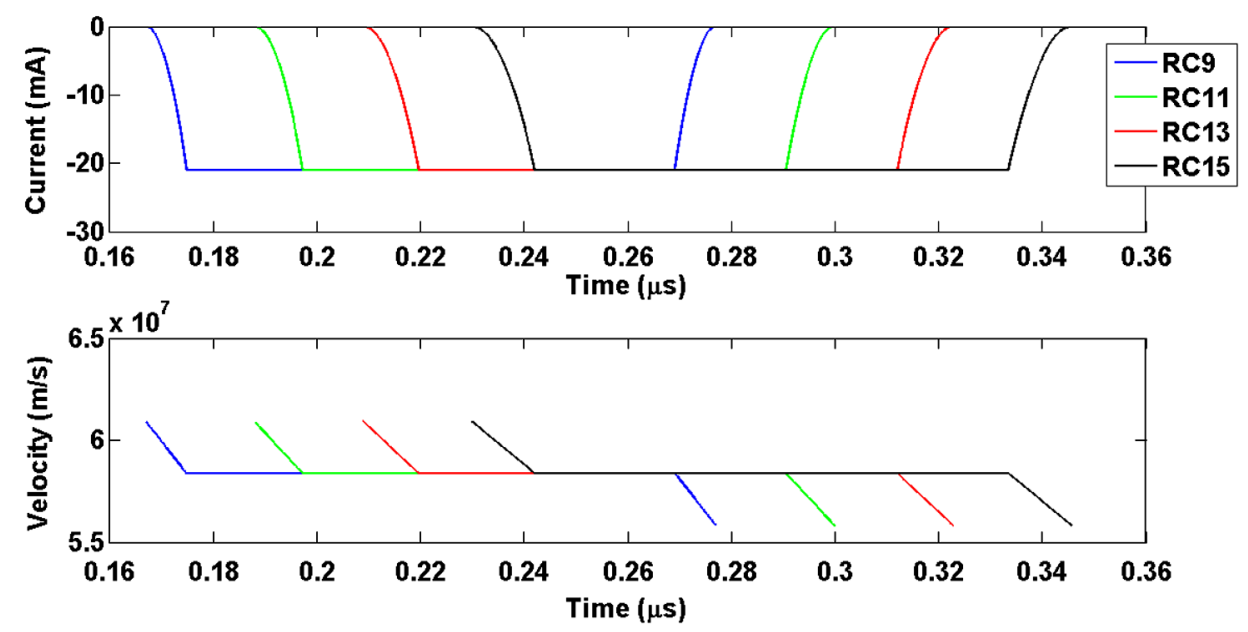

FIG. 3. Analytical current and velocity profiles calculated using the self-similar one-dimensional solution on the first turn, at RC9, $\mathrm{RC} 11, \mathrm{RC} 13$, and $\mathrm{RC} 15$ for the $21 \mathrm{~mA}$ beam.

the beam propagates further around the ring, the head and tail accounts for a larger fraction of the overall bunch length. The head of the beam is $12.1 \mathrm{~ns}$ long at RC15. This is a 55\% increase in length from RC9. Once again, the same is true for the tail of the beam. The maximum velocity at the leading edge of the head is approximately $\mathrm{v}_{\text {head }} \approx \mathrm{v}_{o}+2 c_{s}$, where $\mathrm{v}_{o}$ is the beam velocity [2]. The minimum velocity at the leading edge of the tail is approximately $\mathrm{v}_{\text {tail }} \approx \mathrm{v}_{o}-2 c_{s}$.

Using this one-dimensional model, we can infer the approximate shift in centroid tune at the leading edges of the head and tail of the beam. When the coherent contribution from image forces is included in the calculations, the tune profile becomes asymmetric. This means that the tune shift at the head of the bunch is not equal to the tune shift at the tail of the bunch. This will be shown analytically within this section of the paper and illustrated experimentally in the following section.

In a circular machine, variations in energy or momentum cause a displacement in both the equilibrium orbit through the dispersion function and a shift in the betatron tune through the natural chromaticity $[12,13]$. The natural chromaticity of the lattice $\eta$ is the averaged result of all chromatic aberrations around the machine from pure dipole and quadrupole elements [12]. The chromaticity is used, in this case, to approximate the tune shift at the leading edge of the head as well as the tail. The tune shifts are measured from the leading edge to the midregion of the 


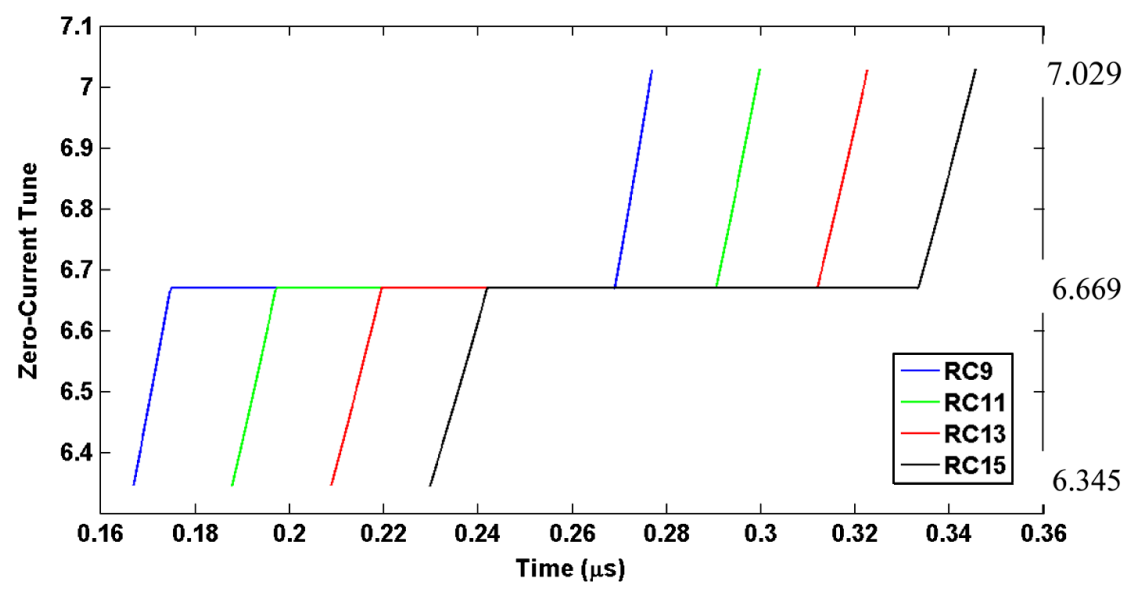

FIG. 4. Analytical zero-current tune profiles calculated on the first turn for the $21 \mathrm{~mA}$ beam, at RC9, RC11, RC13, and RC15.

beam, assuming the beam is divided into three regions (head, midregion, and tail). Using the nominal ring parameters listed in Table I, the analytical longitudinal spacecharge wave velocity is $1.276 \times 10^{6} \mathrm{~m} / \mathrm{s}$ and thus the calculated tune shift at the leading edge of the beam head would be -0.345 using Eq. (1). The tune shift at the leading edge of the beam tail would be +0.345 . The polarity difference is a result of particle acceleration at the beam head and particle deceleration at the beam tail:

$$
\Delta \nu_{\text {head, tail }}=\eta \frac{\Delta p}{p_{o}} \approx \pm \eta \frac{2 c_{s}}{\mathrm{v}_{o}} .
$$

Equation (1) assumes the beam is nonrelativistic. Next, we calculate the analytical zero-current tune profiles at four locations in the ring. The calculations assume no contribution from image forces at this point [4]. The tune dependence on velocity for $N$ focus-drift-defocus-drift (FODO) cell periods in a ring is shown in Eqs. (2) and (3):

$$
\begin{aligned}
& \nu_{o}=\frac{N \sigma_{o}}{2 \pi}, \\
& \nu_{o}=\frac{N}{2 \pi} \cos ^{-1}\left(\cos \theta \cosh \theta+\frac{L}{l} \theta(\cos \theta \sinh \theta\right. \\
& \left.-\sin \theta \cosh \theta)-\frac{1}{2}\left(\frac{L}{l}\right)^{2} \theta^{2} \sin \theta \sinh \theta\right), \\
& \theta=\sqrt{\frac{q B_{o}}{\gamma m a \mathrm{v}_{o}}} l,
\end{aligned}
$$

where $\theta$ represents the focusing strength of the lenses as defined in [8], $l$ the magnet effective length, $L$ the drift space within the FODO cell, and $N$ the total number of FODO cells in the ring [8].

Figure 4 displays the calculated tune profiles at RC9, $\mathrm{RC} 11, \mathrm{RC} 13$, and RC15. The shift in tune at the leading edge of the head is -0.324 and the shift in tune at the leading edge of the tail is +0.360 . Note the positive tune shift at the tail of the beam and the negative tune shift at the head of the beam. This polarity difference corresponds to particle acceleration at the head and deceleration at the tail of the beam. When both magnitudes are averaged, we obtain a result that agrees with the simple chromaticity calculation [using Eq. (1)] resulting in a percent error of $0.87 \%$.

The tune shift induced from transverse space-charge forces has a coherent as well as an incoherent component. The coherent tune shift, due to image forces from a displaced beam in a surrounding pipe [8], can be calculated using Eq. (4):

$$
\Delta \nu_{\mathrm{coh}}=-\left[\frac{1}{(\beta \gamma)^{3}} \frac{1}{I_{o}}\left(\frac{R}{b}\right)^{2}\right] \frac{I_{b}}{\nu_{x o}, \nu_{y o}},
$$

where the peak current is defined by $I_{b}$, the average radius of the machine is defined by $R$, the beam pipe radius is defined by $b$, the relativistic correction is defined by $\beta \gamma$, and the zero-current tune in both $x$ and $y$ is defined either by $\nu_{x o}$ or $\nu_{y o}$, respectively [14]. The coherent component effectively reduces the tune from external quadrupole focusing, by this current dependent effect. Reference [14] describes the measured coherent tune shift, over a wide range of beam currents, in more detail. In this paper, we maintain our focus on the coherent contribution. The calculated values of incoherent tune shift, incoherent tune depression, and beam intensity are listed in Tables I and II of the Appendix for the reader.

Figure 5 displays the calculated tune profiles that include the coherent contribution at RC9, RC11, RC13, and $\mathrm{RC} 15$. The tune at the leading edge of the head is 6.345 and the tune shift is -0.196 . The tune at the leading edge of the tail is 7.029 and the tune shift at the tail is +0.488 .

The coherent contribution is a current dependent effect, which means that the region of the beam with the most current will have the largest contribution. At the leading edges of the beam ends, the tune shift from this contribution also approaches zero as the current approaches zero. When this tune shift is included with the longitudinal space-charge contribution presented, in Fig. 4, it results 


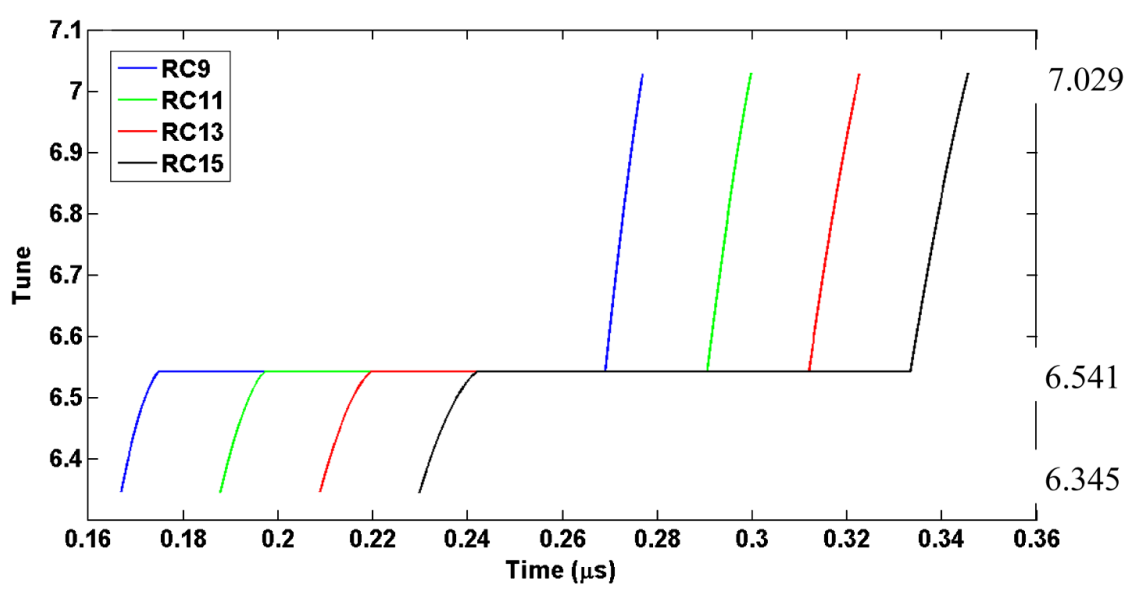

FIG. 5. Analytical tune profile (including coherent contribution) calculated on the first turn for the $21 \mathrm{~mA}$ beam, at RC9, RC11, $\mathrm{RC} 13$, and RC15.

in the asymmetric profile shown in Fig. 5. This is recognized with a smaller tune shift at the beam head and a larger tune shift at the beam tail. The following section presents a diagnostic used to measure the tune profile axially along the bunch, capturing the three regions described above.

\section{BETATRON TUNE PROFILE MEASUREMENTS ALONG A BEAM}

The midregion of the rectangular beam is approximately monoenergetic, thus allowing for various methods to be utilized when measuring tune in that portion of the bunch. Two common methods employed at University of Maryland are the four-turn formula and a fitting method $[12,15]$. The four-turn formula utilizes four turns at a single beam position monitor (BPM) to measure the fractional component of tune. The fitting method utilizes multiple BPMs around known locations on the ring to measure the integer and fractional component of tune. The difficulty with either of these methods when measuring tune outside of the midregion is the fact that the beam ends evolve as the bunch propagates. The path-length dependent evolution of the beam ends impacts the centroid due to the discussion presented in the previous section. This dynamic complicates the measurement methods described above [4].

An alternate method of experimentally measuring tune is to impose small dipole perturbations around the ring, while keeping the measurement point fixed. This is accomplished by measuring the amplitude of the induced shift in betatron phase at a BPM from each of the dipole perturbations [16]. This method, commonly part of the response matrix analysis used for machine optimization [17-19], allows the measurement point to remain fixed while the perturbation point is varied along the ring. Utilizing this method, we were able to avoid the pathlength dependence. The measurement points were also $\mathrm{RC}$, RC11, RC13, and RC15. The perturbed dipoles

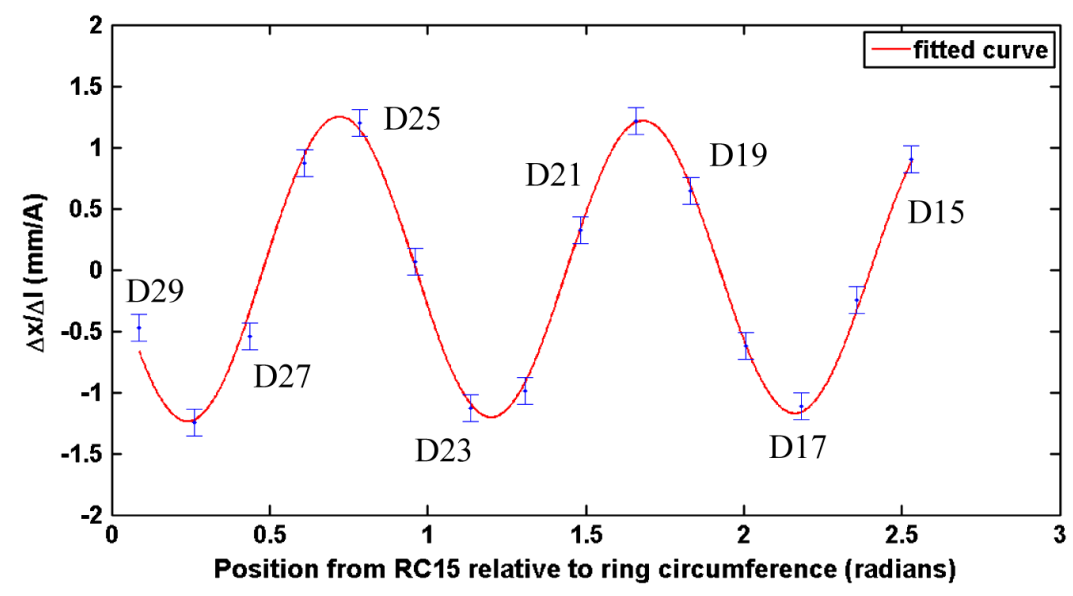

FIG. 6. Measured perturbed horizontal centroid motion (within the midregion of the beam using a 2 ns window) around the ring using a single beam position monitor (BPM at RC15, zero radians). The measured tune is $6.541 \pm 0.062$ with a fit goodness of 0.99 . The average error per point is $0.1 \mathrm{~mm} / \mathrm{A}$. 
were D1 to D29 (where D29 is the last dipole prior to $\mathrm{RC} 15)$. For each chamber, there were 15 dipoles perturbed. This allowed the beam to complete at least two betatron oscillations, obtaining sinusoidal fits with goodnesses greater than or equal to $95 \%$. The goodness is the coefficient of determination or $R^{2}$ of the fit. A perfect fit has a goodness of $100 \%$.

The current in each dipole was varied from $I_{\text {nom }}-$ $0.2 \mathrm{~A}$ to $I_{\text {nom }}+0.2 \mathrm{~A}$ (approximately $\leq 10 \%$ of the nominal current) in $0.05 \mathrm{~A}$ steps, where $I_{\text {nom }}$ is the nominal current. A line was fitted to the data with slope $m=\Delta x / \Delta I$, where $\Delta x$ is the change in transverse position (not equilibrium orbit) from nominal and $\Delta I$ the change in dipole current from nominal. We extracted the tune of the perturbed centroid motion by fitting a sinusoidal function to the slopes measured at each dipole along the length of the accelerator. The circumference of the accelerator is measured in radians ( $\mathrm{RC} 15$ is the location of zero radians). The data, presented in Fig. 6, is taken within the midregion of the bunch averaged over a 2 ns window.

Figure 6 displays the slopes measured at each dipole along the length of the accelerator. The fitted tune (to the data presented in Fig. 6) is $6.541 \pm 0.062$ with a fit goodness of 0.99 . The same measurement obtained identical results at three other chambers. The error bars were obtained by taking the difference between the best fit and the 95\% confidence bounds defined by the fitting algorithms in MATLAB. The error bar of \pm 0.062 is the averaged result measured axially along the bunch.

We then extend this measurement by shifting the $2 \mathrm{~ns}$ window along the axial length of the beam, from head to tail. Shifting the window also extends the response matrix analysis in the longitudinal direction, obtaining betatron tune profiles along the rectangular bunch. Figure 7 displays

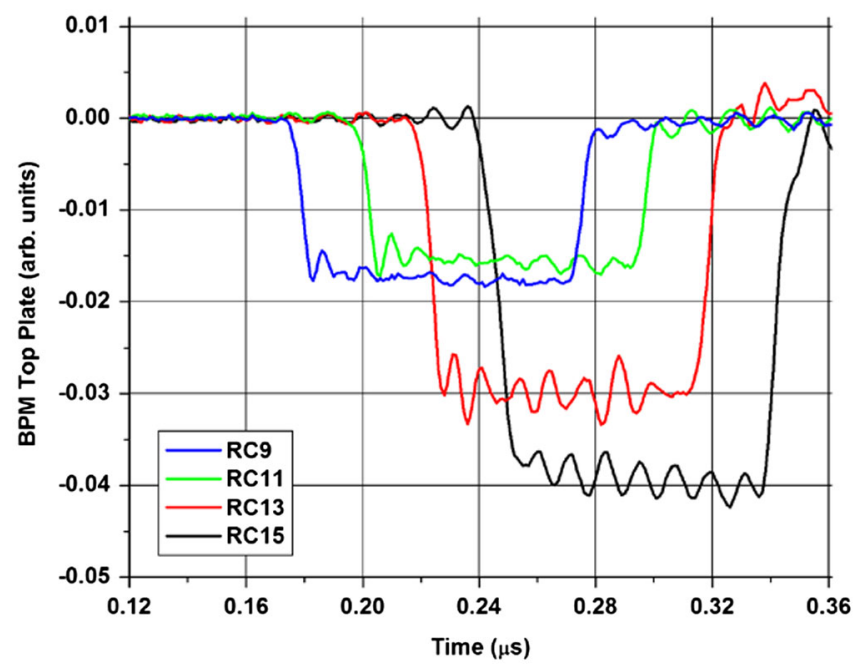

FIG. 7. Top BPM plate at RC9, RC11, RC13, and RC15.

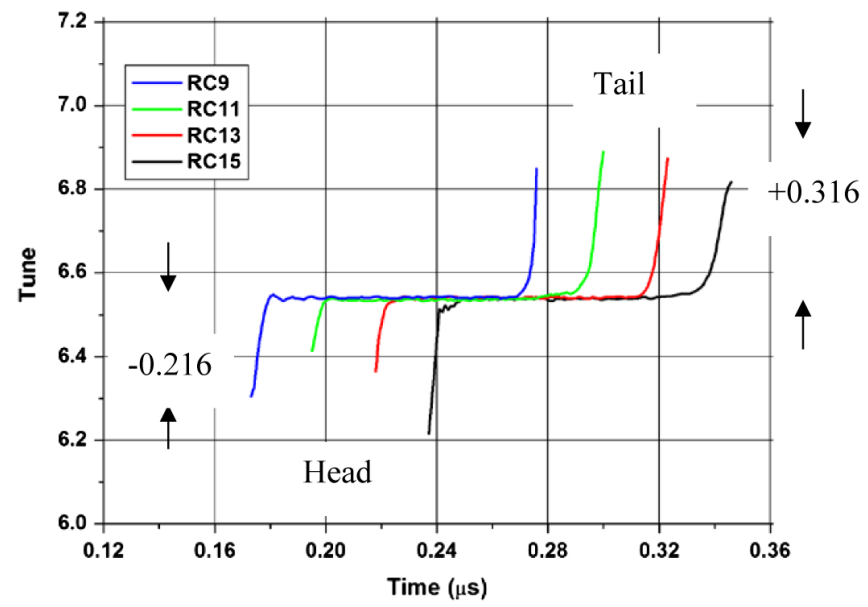

FIG. 8. Horizontal tune as a function of beam length, measured using a single beam position monitor at RC9, RC11, RC13, and RC15.

a single BPM plate as a time-to-beam correlation for the tune profile comparisons.

Comparisons of bunch head and tail lengths (shown in Fig. 7) with calculations presented in the previous section indicate that the agreement at RC9 has a percent error of $3.8 \%$. Comparisons at RC15 indicate a percent error of $11.6 \%$. We suspect that the disagreement with measurements at RC15 is due to noisy BPM electronics. This is in contrast to the less noisy BPM at RC9.

Figure 8 displays the tune profile measured at RC9, $\mathrm{RC} 11, \mathrm{RC} 13$, and RC15. As presented in Sec. II, the tune decreases at the head of the beam (shown in Fig. 8) and increases at the tail. The head tunes average over the four ring chambers is $6.325 \pm 0.062$, and the average of the tail tunes is $6.857 \pm 0.062$. This corresponds to a -0.216 shift in tune at the head of the beam and a +0.316 shift at the tail. The measurements agree with the computed values presented in Sec. II that include the coherent contribution, resulting in a percent error of $0.32 \%$ and $2.45 \%$ for the head and tail, respectively.

The measurement technique has also shown to be robust enough to resolve the tune in laboratory environments with noise on most BPMs (as illustrated with the $\sim 100 \mathrm{MHz}$ signal on all the BPM plates). The following section presents a destructive first turn diagnostic that resolves the head and tail cork-screwing motion at the beam ends.

\section{SLICED PHOSPHOR SCREEN MEASUREMENTS ALONG A BEAM}

As discussed in Sec. II, longitudinal space charge alters the energy at the beam ends when no longitudinal confining fields are used $[4,11,20,21]$. Ring dispersion then causes a centroid displacement that is correlated axially along the length of the bunch [22]. In order to image 

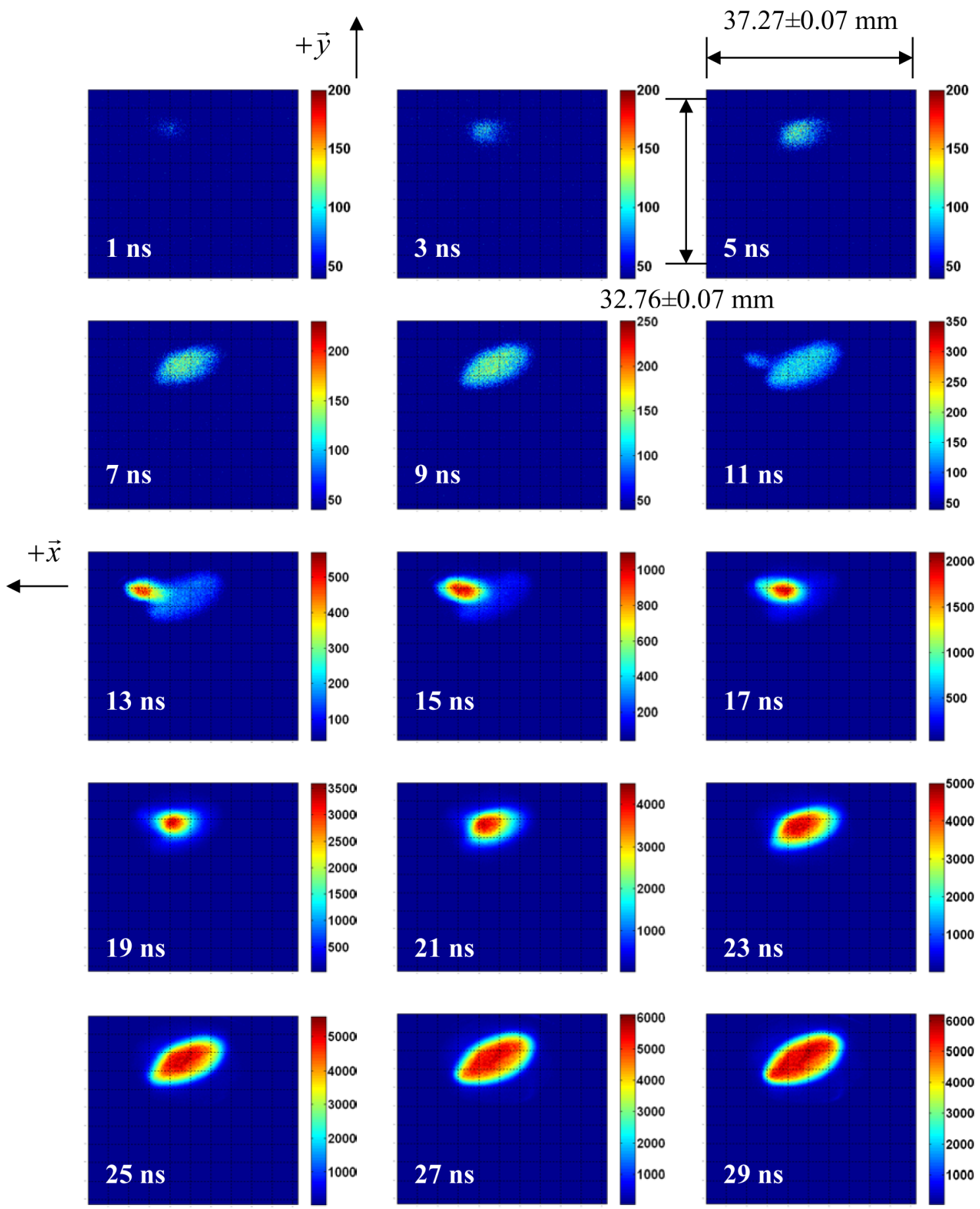

FIG. 9. 3 ns gated camera images of the $21 \mathrm{~mA}$ beam head, measured at RC15 as a function of time along the beam pulse.

this motion, we use a combined 2.4 ns phosphor screen and 3 ns gated 16-bit PIMAX2 ICCD camera installed at RC15. This allows us to measure the transverse beam displacement as a function of axial position along the bunch. This diagnostic is able to sequentially resolve 3 ns sliced images. Figures 9 and 10 display the results measured on the RC15 phosphor screen for the head and tail, respectively. The integrated camera images are sequentially delayed by $2 \mathrm{~ns}$ axially along the bunch, beginning just before the beam and ending just after. The measured head and tail lengths are approximately a factor of 5 longer than the sliced resolution on the first turn.

The beam head exhibits a cork-screwing motion of the charge distribution (shown in Fig. 9) over the consecutive sliced images. 


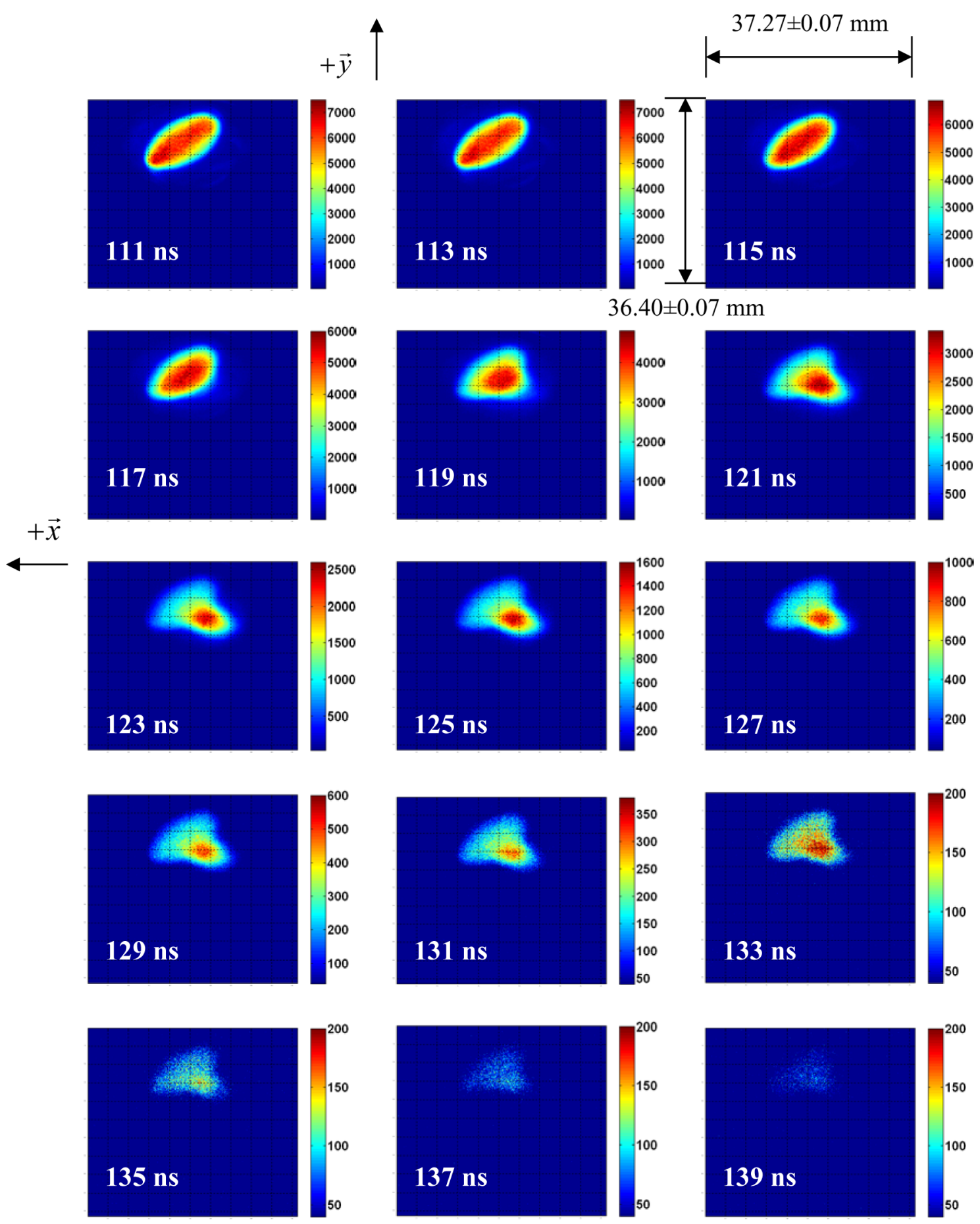

FIG. 10. $3 \mathrm{~ns}$ gated camera images of the $21 \mathrm{~mA}$ beam tail, measured at RC15 as a function of time along the beam pulse.

The tail of the beam also exhibits a similar movement of the charge distribution. A calibrated transverse centroid measurement is shown in Fig. 11.

The maximum overall horizontal and vertical displacement caused by the beam head and tail regions is $6.00 \pm 0.07$ and $2.21 \pm 0.07 \mathrm{~mm}$, respectively. The blue points (shown in Fig. 11) at the origin are the movement of the beam midregion by $0.64 \pm 0.07 \mathrm{~mm}$. The centroid measurement clearly displays the cork-screwing motion at the ends of the beam, illustrated by the green and red loops, for the head and tail, respectively. This centroid motion results when an off equilibrium particle, in both the horizontal and vertical plane, performs a helical motion as different particle energies are sampled axially along the bunch. The motion is resolvable at one diagnostic location due to the electronic delay imposed with the measurement 


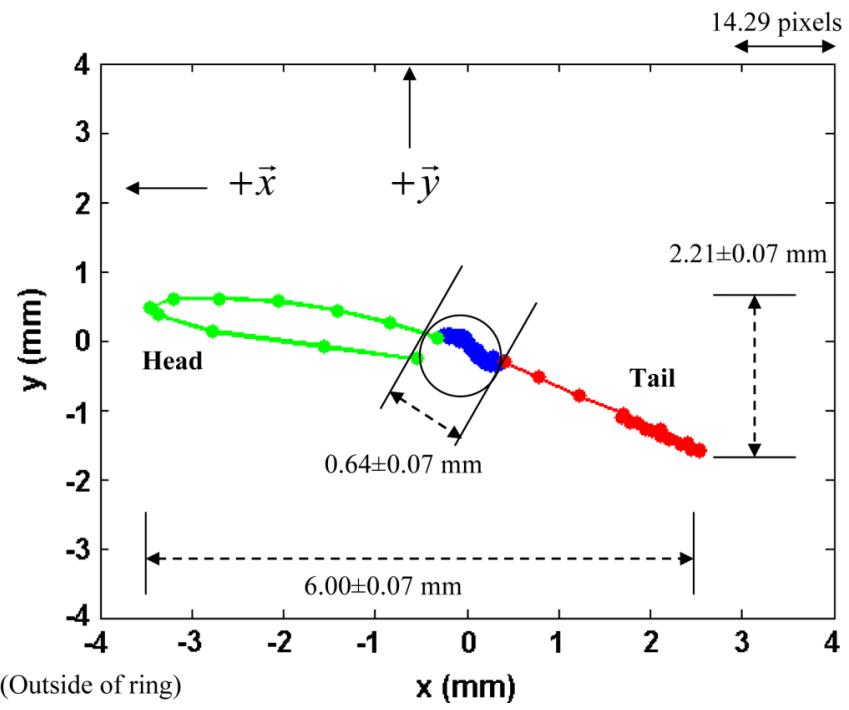

FIG. 11. Centroid measurements from the 3 ns gated camera images of the $21 \mathrm{~mA}$ beam, measured as a function of beam length at $\mathrm{RC} 15$. We subtract the position where $(0,0)$ refers to the centroid of the beam center from all data points. (Calibration was $0.07 \mathrm{~mm} /$ pixel in both $x$ and $y$.)

apparatus. This measurement technique allows us to optically sample different axial slices (or different energies) along the bunch. In the case of the beam described in this paper, were able to resolve the three distinct regions of the bunch (head, midregion, tail).

\section{CONCLUSION}

We have presented two methods that use the ring characteristics to give insight into the energy evolution of the beam ends of a space-charge dominated beam. The first method is an extension of the nondestructive response matrix diagnostic, which resolves a tune profile axially along the bunch. The tune shift measured at the head of the beam was -0.216 and the tune shift at the tail of the beam was +0.316 . Comparisons with calculations that include the coherent contribution from image forces agree with a percent error of $0.32 \%$ and $2.45 \%$ for the head and tail, respectively. The asymmetry in the head and tail tune shifts illustrates the summation of both contributions from longitudinal space charge and that from transverse image forces. The second method is a first turn destructive diagnostic that is used to optically resolve the "cork-screwing" motion axially along the bunch, at a fixed location (RC15). The measurement displays the centroid motion that results when an off equilibrium particle, in both planes, performs a helical motion as different particle energies are optically sampled axially along the bunch.

Extending the first method to measure these profiles over multiple turns (using fast pulsed dipoles) would also give us optimization information for longitudinal containment. This could be implemented using a fast turn-by-turn adaptive wideband rf system, which would create the corrective longitudinal fields to reduce the energy gained at the head of the bunch and increase the energy lost at the tail of the bunch $[2,4]$. As pointed out in Sec. II, the longitudinal dynamics of the bunch is current dependent and changes with current loss during propagation over many turns [2]. When operating UMER as a storage ring, the rf system used to contain the bunch must also evolve with the loss rate. This is necessary to avoid the excitation of space-charge waves at the beam edges, as has been shown in Ref. [2].

\section{ACKNOWLEDGMENTS}

We wish to acknowledge Hao Zhang and Ralph Fiorito for assistance with the imaging system and Kamal Poor Rezaei for the improved steering. We would also like to acknowledge I. Haber, M. Cornacchia, D. F. Sutter, T. W. Koeth, and S. Bernal for their helpful discussions. This work is supported by the U.S. Department of Fusion Energy Science and Energy High Energy Physics and by the Department of Defense Office of Naval Research and Joint Technology Office.

\section{APPENDIX}

TABLE I. UMER main ring parameters.

\begin{tabular}{|c|c|c|c|c|c|}
\hline Beam energy (keV) & $\beta=\mathrm{v} / c$ & $\begin{array}{l}\text { Initial pulse } \\
\text { length (ns) }\end{array}$ & $\begin{array}{l}\text { Average ring } \\
\text { radius }(\mathrm{m})\end{array}$ & $\begin{array}{l}\text { Lap time } \\
\quad(\mathrm{ns})\end{array}$ & $\begin{array}{c}\text { Repetition } \\
\text { rate }(\mathrm{Hz})\end{array}$ \\
\hline 9.967 & 0.19467 & 100.82 & 1.8335 & 197.39 & 60.0 \\
\hline FODO period $(\mathrm{m})$ & $N$ FODO Periods & $\begin{array}{c}l \text {-magnet effective } \\
\text { length }(\mathrm{m})\end{array}$ & $\begin{array}{c}L \text {-drift } \\
\text { space }(\mathrm{m})\end{array}$ & $\begin{array}{l}\text { Zero-current } \\
\text { tune } \nu_{o}\end{array}$ & $\begin{array}{c}\text { Peak gradient } \\
(\mathrm{G} / \mathrm{cm} / \mathrm{A})\end{array}$ \\
\hline 0.32 & 36 & 0.0447 & 0.16 & 6.669 & 3.8019 \\
\hline
\end{tabular}


TABLE II. Parameters for a beam with a current of $21 \mathrm{~mA}$.

\begin{tabular}{lccccc}
\hline \hline $\begin{array}{l}\text { Beam current } \\
(\mathrm{mA})\end{array}$ & $\begin{array}{c}\text { Beam radius } \\
(\mathrm{mm})\end{array}$ & $\begin{array}{c}\text { Pipe radius } \\
(\mathrm{mm})\end{array}$ & $\begin{array}{c}\text { Initial emittance } \\
(\mathrm{mm}-\mathrm{mr})\end{array}$ & $\begin{array}{c}\text { Incoherent } \\
\text { tune shift }\end{array}$ & $\begin{array}{c}\text { Incoherent tune } \\
\text { depression }\left(\nu / \nu_{o}\right)_{\text {inc }}\end{array}$ \\
\hline 21.0 & 5.142 & 25.4 & 30.23 & -4.57 & 0.314 \\
\hline \hline
\end{tabular}

Intensity parameter

$\chi=1-\left(\nu / \nu_{o}\right)_{\text {inc }}^{2}$

Coherent tune shift

$g$ factor

Natural chromaticity $\eta$

Dispersion $(\mathrm{cm})$

\begin{tabular}{lllll}
0.901 & -0.128 & 3.154 & -7.9 & 4.6 \\
\hline \hline
\end{tabular}

[1] M. Shocket, Symmetry Magazine 06, 34 (2009).

[2] B. Beaudoin, I. Haber, R. A. Kishek, S. Bernal, T. Koeth, D. Sutter, P. G. O’Shea, and M. Reiser, Phys. Plasmas 18, 013104 (2011).

[3] W. M. Sharp, D. A. Callahan, and D.P. Grote, in Proceedings of the 8th International Committee for Future Accelerators Workshop, 1995.

[4] B. L. Beaudoin, Ph.D. thesis, University of Maryland, 2011.

[5] A. Friedman, J. J. Barnard, R. J. Briggs, R. C. Davidson, M. Dorf, D. P. Grote, E. Henestroza, E. P. Lee, M. A. Leitner, B. G. Logan, A. B. Sefkow, W. M. Sharp, W. L. Waldron, D. R. Welch, and S.S. Yu, Nucl. Instrum. Methods Phys. Res., Sect. A 606, 6 (2009).

[6] E. Voorhies, R. A. Kishek, T. Koeth, S. Bernal, P. G. O'Shea, and I. Haber, in Proceedings of the 2011 Particle Accelerator Conference, NY, USA (IEEE, New York, 2011).

[7] A. Faltens, E. P. Lee, and S. S. Rosenblum, J. Appl. Phys. 61, 5219 (1987).

[8] M. Reiser, Theory and Design of Charged Particle Beams (Wiley-VCH Inc., Weinheim Germany, 2008), 2nd ed., pp. 153-156, 271, 399, 498-515.

[9] L. Smith, ERDA Summer Study for Heavy Ion Inertial Fusion, edited by R. O. Bangerter, W. B. Herrmannsfeldt, D. L. Judd, and L. Smith (Lawrence Berkeley Laboratory, Berkeley, 1976), pp. 77-79.

[10] A. Hofmann, in Proceedings of the International School of Particle Accelerators, Erice, Italy, 1976, edited by M. H. Blewett, A.Z. Zichichi, and K. Johnsen (CERN, Geneva, 1977), p. 139.

[11] Y. Cui, Y. Zou, M. Reiser, R. A. Kishek, I. Haber, S. Bernal, and P. G. O'Shea, Phys. Rev. ST Accel. Beams 7, 072801 (2004).
[12] P. J. Bryant and K. Johnsen, The Principles of Circular Accelerators and Storage Rings (Cambridge University Press, Cambridge, England, 1993), p. 120-122.

[13] H. Wiedemann, Particle Accelerator Physics II (SpringerVerlag, New York, 1993), 2nd ed., p. 397.

[14] D. Sutter, B. L. Beaudoin, S. Bernal, M. Cornacchia, R. A. Kishek, T. Koeth, P. G. O'Shea, and M. Reiser, in Proceedings of the 2011 Particle Accelerator Conference, NY, USA (Ref. [6]), WEP102.

[15] D. Sutter, S. Bernal C. Wu, M. Cornacchia, B. Beaudoin, K. Fiuza, I. Haber, R. A. Kishek, M. Reiser, and P. G. O'Shea, in Proceedings of the 23rd Particle Accelerator Conference, Vancouver, Canada, 2009 (IEEE, Piscataway, NJ, 2009), FR5PFP063.

[16] R. A. Kishek, UMER Technical Note No. UMER-100505-RAK, 2010.

[17] J. Safranek, Nucl. Instrum. Methods Phys. Res., Sect. A 388, 27 (1997).

[18] J. Safranek, "International Committee for Future Accelerators," Beam Dynamics Newsletter No. 44, edited by W. Chou. and A. Ghodke, 2007.

[19] V. Sajaev, V. Lebedev, V. Nagaslaev, and V. Valishev, in Proceedings of the 21st Particle Accelerator Conference, Knoxville, 2005 (IEEE, Piscataway, NJ, 2005).

[20] D. Neuffer, IEEE Trans. Nucl. Sci. 26, 3031 (1979).

[21] D. X. Wang, J. G. Wang, and M. Reiser, Phys. Rev. Lett. 73, 66 (1994).

[22] S. Bernal, B. Beaudoin, T. Koeth, and P. G. O'Shea, Phys. Rev. ST Accel. Beams 14, 104202 (2011). 